\title{
Kernos
}

Revue internationale et pluridisciplinaire de religion grecque antique

$17 \mid 2004$

Varia

\section{Pierre BONNECHERE, Trophonios de Lébadée. Cultes et mythes d'une cité béotienne au miroir de la mentalité antique}

Vinciane Pirenne-Delforge

\section{(2) OpenEdition}

\section{Journals}

\section{Édition électronique}

URL : http://journals.openedition.org/kernos/1503

DOI : 10.4000/kernos. 1503

ISSN : 2034-7871

\section{Éditeur}

Centre international d'étude de la religion grecque antique

\section{Édition imprimée}

Date de publication : 1 janvier 2004

ISSN : 0776-3824

\section{Référence électronique}

Vinciane Pirenne-Delforge, «Pierre Bonnechere, Trophonios de Lébadée. Cultes et mythes d'une cité béotienne au miroir de la mentalité antique », Kernos [En ligne], 17 | 2004, mis en ligne le 16 juin 2011, consulté le 22 septembre 2020. URL : http://journals.openedition.org/kernos/1503 ; DOI : https:// doi.org/10.4000/kernos.1503 
à notre connaissance de la mentalité antique un traitement des images mythologiques qui ne pose pas le problème de cette dimension-là?

Cornelia Isler-Kerényi

(Zurich, Archäologisches Institut der Universität)

BONNECHERE Pierre, Tropbonios de Lébadée. Cultes et mythes d'une citê béotienne au miroir de la mentalité antique, Leiden, Brill, 2003. 1 vol. $16 \times$ $24,5 \mathrm{~cm}, \mathrm{xxX}+430 \mathrm{p}$. (Religions in the Graeco-Roman World, 150). ISBN : 9004-13102-7.

Trophonios est un personnage somme toute assez secondaire de la mythologie grecque, à la fois architecte et devin, fils d'Apollon ou d'un mortel selon les cas. Le caractère exceptionnel de son culte béoticn est donc remarquable au vu de cette relative "inconsistance » mythologique : le consultant de l'oracle vivait une expérience hors du commun, dont Plutarque et Pausanias - on reviendra sur ces témoignages nous ont laissé des tableaux haut en couleur. Le dossier qui est traité dans ce livre est plus qu'exhaustif : il exploite absolument tous les témoignages qui, de près ou de loin, permettent d'éclairer la figure du héros/dieu et les pratiques mantiques liées à son sanctuaire de Lébadée. Il s'agit donc d'une somme remarquable, qui restera longtemps un passage obligé pour quiconque veut aujourd'hui " interroger » Trophonios.

Une brève description des articulations du livre tout d'abord. La $1^{\text {re }}$ partie pose le cadre : le site de Lébadée est minutieusement décrit, avec un aperçu préliminaire des rites. La $2^{\star}$ partie traite de la «mythologie trophoniaque», c'est-à-dire des quelques allusions au personnage dans la littérature grecque, et surtout des figures particulières auxquelles il est apparenté dans des listes thématiques, notamment chez Strabon : des devins comme Amphiaraos et Amphilochos, des poètes comme Orphée et Musée, des "chamans» comme Zalmoxis ou même Empédocle et des héros tels Aristée ou Asclépios. Le statut de Trophonios est donc ambigu : entre héros et daimôn, toujours un peu à la lisière de la nature divine. La $3^{\circ}$ partie offre une première analyse des modalités de la révélation trophoniaque, après avoir posé l'hypothèse de la dimension 'mystérique' du rituel : on y voit un consultant psychiquement affaibli et soumis à des préparatifs qui vont favoriser l'émergence d'un "état modifié de conscience ", selon nos catégories modernes. En termes grecs, une telle expérience est vue comme une échappée de l'âme hors du corps, ainsi que Plutarque l'interprète (mais pas Pausanias). C'est donc entre oracle et mystère que se placerait la descente dans l'adyton de Lébadée, des rapprochements avec les données éleusiniennes refermant cette partie. La $4^{\prime \prime}$ partie entre davantage dans le détail des rites proprement dits (l'alsos comme lieu de passage, préparations diverses et variées, accès à une révélation, remémoration, récupération progressive du rire, etc.), pour les replacer ensuite sur l'arrière-plan plus large des mouvements religieux «marginaux » comme le pythagorisme et l'orphisme. La $5^{"}$ partie livre une étude de ce que l'on sait du « panthéon » lébadéen, afin d'insérer la figure de Trophonios dans le contexte divin local auquel il appartient. Une substantielle conclusion boucle le parcours, avant les sept annexes qui sont surtout des tableaux reprenant les données des sources de manière synoptique. Une riche bibliographie et un index thématique referment l'ensemble. Manque toutefois un index des sources.

Comme souvent lorsqu'on étudie les données religieuses grecques, le témoignage de Pausanias est fondamental dans ce dossier - et tout le passage du livre IX (39, 1 $40,2)$ est présenté par l'A. en tête de son ouvrage, avec une traduction. Le Périégète a consulté l'oracle de Trophonios et il décrit le parcours auquel tout impétrant était 
soumis. L'expérience devait être éprouvante, pour ne pas dire traumatisante, et Pausanias conclut en disant que chaque consultant devait dédier une tablette mentionnant tout ce qu'il avait vu ou entendu. Plutarque est l'autre référence essentielle. Dans le De genio Socratis, surtout, Timarque reçoit dans l'antre trophoniaque la révélation de l'organisation du cosmos et de la vie. La démonologie de l'auteur est évidemment essentielle dans ce traité. Il fait de Trophonios un des daimones bienveillants à l'origine des révélations mantiques, ce qui ne l'empêche pas de le qualifier de theos! Timarque se trouve dans un état à la limite entre sommeil onirique et vision éveillée, ce qui correspond bien à la transe ou à la syncope propre à l'état modifié de conscience. C'est un point sur lequel on suit passionnément les développements de l'A., même si la dimension "chamanique » de l'expérience ne laisse pas d'être problématique. L'A. cherche à se positionner par rapport à un courant interprétatif qui a usé et abusé de la notion et jamais les guillemets ne sont oubliés quand apparaissent les termes de «chaman» et de «chamanisme». La prudence est donc d'application. Cependant, si l'on voit plus ou moins comment des figures telles Aristéas ou Empédocle ont pu être interprétées en termes «chamaniques», Trophonios a-t-il vraiment le profil ? Le seul élément de sa biographie légendaire exploitable en ce sens est l'engloutissement dans la terre et, selon l'A., son statut de "passeur" vers les Enfers. En fait, c'est l'expérience même du consultant qui est ainsi projetée sur le dieu qui la pattonne, mais a-t-on véritablement progressé dans la compréhension du vécu de ces gens (par ailleurs bien décrit en termes d'EMC) en l'étiquetant de la sorte? Il aurait peut-être été préférable d'évacuer d'emblée - et brièvement - la dimension "chamanique" du champ de l'investigation plutôt que de faire apparaître une telle notion ça et là dans le discours - fût-ce en l'assortissant de guillements.

La relation entre révélation oraculaire et révélation «mystérique » est l'autre propos central du livre. C'est même un des fils conducteurs d'une démonstration qui entend ne négliger aucun élément pour asseoir la conviction du lecteur. Il s'agit en fait de montrer comment un nombre impressionnant d'éléments intervenant dans la consultation trophoniaque entrent en résonance avec ce que l'on sait par ailleurs des mystères grecs au sens large, d'Éleusis à l'orphisme et au pythagorisme: purficications diverses, alimentation particulière, vêtements spécifiques - en ce compris les chaussures -, sacrifices, mise en situation psychologique extrême, perte du rire, eaux de la Mémoire et de l'Oubli, trône de la remémoration. La convergence des clonnées est impressionnante et il devient évident, au fil de la démonstration, que l'expérience vécue par le consultant à Lébadée avait une "coloration mystérique ». Elle remonte au moins à la période classique puisque les Nuées d'Aristophane associent parodiquement une expérience de type éleusinien à la figure de Trophonios pour décrire «l'initiation " de Strepsiade au "pensoir» de Socrate. La consultation oraculaire à Lébadée peut donc être comparée à une démarche initiatique, au sens où il s'agit d'une expérience dramatisée autour d'une révélation qui est plus existentielle qu'intellectuelle (le pathein plutôt que le mathein). Comparaison, oui, assimilation, non, même si la frontière n'est pas toujours totalement claire, ni dans les données antiques ni, dès lors, dans la démonstration de l'A. Il y a en effet deux différences de taille entre ce que les Grecs nommaient teletè et le manteion béotien. D'une part, la «révélation " dont le consultant est gratifié dans l'adyton est intimement liée à son état mental personnel et à ses propres attentes - c'est en cela qu'il s'agit bien d'une consultation oraculaire - et non au "profil » du dieu qui patronne la révélation, comme dans le cas des mystères démétriaques ou bachiques. Il ne s'agit pas d'exclure que les teletai aient pu atteindre, au-delà du "message », quelque chose d'autre, davantage lié à l'expérience personnelle de l'initié, mais s'il y a des cultes à mystères patronnés par des dieux différents, 
c'est bien que les messages devaient l'être aussi. D'autre part, la dimension du secret est totalement absente à Lébadée. Bien au contraire, il existe une obligation stricte de consigner son expérience par écrit et de l'exposer au vu et au su de tous les visiteurs du sanctuaire. Il est donc excessif de conclure (p. 331) que Trophonios fait partie de ces figures « détentrices de secrets mystériques », comparables aux « chamans » grecs.

La comparaison entre Lébadée et les teletai a donc été instruite «à charge » et non "à décharge »: les similitudes sont privilégiées - et il faut reconnâitre qu'elles sont nombreuses et souvent méconnues - aux dépens des différences que l'on vient de souligner. Or ce sont probablement ces différences qui permettent de comprendre la portée d'une des sources principales de ce dossier, à savoir le texte de Pausanias. En effet, dans l'usage de son vocabulaire «technique ", l'auteur de la Périégèse est strict et cohérent. Pourtant, à aucun moment, il n'use du terme teletê pour désigner le culte de Trophonios. Seul manteion est apte à l'évoquer. Étant donné le caractère central de ce passage dans la démonstration, il eût peut-être fallu tenir davantage compte de la portée de ce type de vocabulaire dans la Périégèse. Le mot teletè y apparaît fréquemment, mais toujours lié à un interdit. Pour Pausanias, donc, la consultation oraculaire est certes une expérience exceptionnelle, mais une teletè, c'est autre chose, qui le cantonne la plupart du temps dans un mutisme respectueux. Or, quand il parle des "colorations orphiques et pythagoriciennes» (p. 290) du sanctuaire béotien, l'A. souligne vivement que l'absence de référence à ce type de courant chez Pausanias n'est due "ni à un oubli ou un manque de perspicacité du Périégète... ", mais à l'appartenance du sanctuaire à ce vaste ensemble religieux qui avait aussi donné naissance à l'orphisme et au pythagorisme. Que dire alors de l'absence de référence à une télétè ? Ni oubli ni manque de perspicacité... Pour les pèlerins qui s'y rendent, Lébadée est avant tout un sanctuaire oraculaire. Mais il faut rendre grâce à P. Bonnechere d'avoir montré que le contact direct avec la divinité intervenant dans ce rituel traumatisant et les préparatifs qu'il requiert sont les éléments-clés qui justifient le rapprochement opéré par les auteurs anciens eux-mêmes entre Trophonios et les mystères. Il ne s'agit pas uniquement des tendances «syncrétiques» de la période impériale. En outre, «tout au long de son histoire, le sanctuaire de Lébadée a constitué pour les penseurs une espèce de laboratoire où ils pouvaient tenter d'appliquer diverses approches philosophiques au problème de la révélation " (p. 282). Cette potentialité très grande de la révélation trophoniaque - en quoi c'est avant tout un sanctuaire oraculaire, mais sans intermédiaire - permet de comprendre que nos cleux sources principales, dont les auteurs sont presque contemporains, aient pu témoigner à des niveaux de " conscience religieuse " - ou plutôt philosophique ? - très différents.

En fait, ce qui unit mystères, oracles et cultes médicaux, outre une certaine expérience du contact avec la divinité, c'est le « dévoilement » de quelque chose d'ordinairement caché ou incompris (fautes du passé, événements à venir, eschatologie...). Ce qui les différencie, c'est que, en contexte mystérique, un tel clévoilement est lié à une forme de message - le fameux hieros logos - plus ou moins précis laissé aux hommes par la divinité ou la figure héroïque «maîtresse des mystères » et fait l'objet d'un secret. Ce n'est le cas ni en contexte oraculaire, ni médical.

Ces réflexions critiques montrent tout l'intérêt du travail et ne doivent pas occulter les nombreux développements qui témoignent d'une excellente connaissance de la pensée religieuse des Grecs, de la mâitrise de tous types de sources et d'une grande finesse d'analyse. Le principal défaut du livre est sans doute son foisonnement, manifeste dans les méandres du parcours - notamment l'émiettement du propos en quantité de sous-sections. Le mieux est parfois l'ennemi du bien et la volonté de ne 
rien négliger finit par être contre-productive. La conclusion du livre, très fournie, est donc bienvenue et permet de renouer tous les fils de la pelote patiemment dévidée.

Vinciane Pirenne-Delforge

(FNRS - Université de Liège)

Michel Mazoyer, Télipinul, le dieu au marécage. Essai sur les mythes fondateurs du Royaume bittite, Paris, L'Harmattan, 2003. 1 vol. $14 \times 22 \mathrm{~cm}, 393 \mathrm{p}$. (coll. KUBABA, Série Antiquité II), ISBN : 2-7475-4345-5.

Télipinu, le dieu hatti de l'agriculture, est connu par un certain nombre de textes mythologiques et de rituels. L'A. les a rassemblés et en fournit une translittération, une traduction ainsi qu'un commentaire grammatical et lexical; en y ajoutant les mythes parallèles qui évoquent les dieux disparus (mythes de la «Disparition du Soleil», de “Télipinu et la fille de l'Océan » et de la «Disparition du dieu de l'Orage de Nerik »), il démêle la succession des récits mettant en scène des dieux "boudeurs", dieux qui partagent la caractéristique de disparaître et de reparaître plus tard. En comparant d'une part les thèmes exposés dans le mythe de Télipinu et dans les mythes parallèles et, d'autre part, le ductus et la langue de chacun de ces textes, l'A. propose de dater la rédaction de la première version du mythe de Télipinu du règne du roi-fondateur hittite homonyme, Télipinu, vers 1525-1500 av. n. è. La thèse autour de laquelle l'ouvrage s'articule est que, dieu agraire fils du dieu de l'Orage emprunté par les Hittites au fonds religieux hatti, Télipinu serait devenu, sous l'influence des «envahisseurs » indo-européens l'un des dieux les plus importants du panthéon, un dieu fondateur, en l'honneur duquel ce texte mythologique aurait été composé. Pour rappel, en raison d'une faute commise envers lui, Télipinu quitte son sanctuaire et se réfugie dans un marécage où il s'endort. Tout dépérit en son absence. Après des tentatives infructueuses menées par diverses personnalités du panthéon, une abeille envoyée par la déesse magicienne Kamrušepa retrouve le dieu, le réveille en le piquant puis le purifie de la colère dans laquelle un réveil aussi brutal l'a plongé par une série de rituels relevant de la magie analogique; finalement le dieu apaisé rentre dans son temple, ramenant avec lui la fertilité et la fécondité et plante devant le palais royal l'arbre qui le symbolise, auquel il accroche la karša, sac en peau animale censée contenir toutes les richesses. Ainsi fait-il du couple royal le dépositaire de ce précieux bagage et institue-til la royauté hittite.

Si jusqu'à présent on s'accordait à voir dans le mythe de Télipinu l'expression du cycle agraire, l'originalité de la thèse de l'A. est de montrer qu'au cours du mythe, Télipinu évolue, se comportant encore au début du récit comme un dieu agraire anatolien, mais à la fin, doté d'une nouvelle fonction, comme un dieu hittite. Une série d'éléments repérables dans les différentes versions du mythe seraient chargés de conférer à Télipinu un caractère de dieu fondateur de royaume. Parmi ces traits, l'A. observe notamment qu'à son retour son premier geste est de mettre en état l'autel sacrificiel et qu'il légitime le futur fondateur humain. Apportant de surcroît au roi le respect, la gloire et la soumission pour l'ensemble du royaume, il assure une descendance au couple royal; sa fondation, il la protège par ses bonnes relations avec les divinités protectrices (les " $L A M M A$ ) de même qu'il protège les limites du territoire. Mais l'A. va plus loin en comparant ces éléments avec le comportement de l'Apollon Archégète qui, lui aussi, accomplit une série de gestes de fondateur dans des circonstances analogues: Apollon remet en état l'autel sacrificiel, légitime le fondateur humain, assure la soumission des sujets et des alliés, s'associe au processus de renouvellement des générations et protège le territoire de la cité. De ce parallélisme nou- 